\title{
Patrones diarios de actividad de la hormiga Azteca constructor (Hymenoptera: Formicidae) y su relación con la presencia de alimento
}

\author{
Pablo R. Gutiérrez-Martínez y Diana Acuña-Sánchez \\ Escuela de Biología, Universidad de Costa Rica, 2060 San José, Costa Rica; pablorgmbiol@hotmail.com, diana.ucr86@gmail.com
}

Recibido 18-III-2013 Corregido 1-V-2013 Aceptado 25-V-2013

\begin{abstract}
Daily activity patterns of the ant Azteca constructor (Hymenoptera: Formicidae) and their relationship with food presence. Ant activity shows rhythmic variations that suggest a chrono-biological integrated system or a daily rhythm synchronized with the Earth's geophysical variations. Previous studies have shown that the activity of ant colonies can vary between seasons and along the day, which in some cases relates to food supply. We recorded the daily activity of Azteca constructor in the field and the laboratory and found no correlation with the presence of Müllerian food bodies. Captive colonies increased their activity around midday, suggesting a daily cycle. Müllerian body production increased with increasing ant activity. The relationship between Pseudococcidae and Azteca ants made us doubt the benefits received by the plant in its relationship with Azteca. Finally, the activity of colonies in the field varied widely, suggesting that several environmental factors influence ant activity.
\end{abstract}

\section{KEY WORDS}

Pseudococcidae, Müllerian bodies, trichilium, daily rhythm, photoperiod

\section{RESUMEN}

La actividad de las hormigas, al igual que en el resto de los seres vivos, presenta variaciones rítmicas que sugieren un sistema cronobiológico integrado o ciclo diario sincronizado con las variaciones geofísicas de la Tierra. Estudios previos en hormigas han demostrado que la actividad de las colonias puede variar entre estaciones, a lo largo del día y a la oferta de alimento de la planta a la que la colonia se encuentra vinculada. El presente trabajo tiene como objetivos registrar los patrones de actividad diaria de Azteca constructor en condiciones de cautiverio y en el campo, y si su actividad varía ante la presencia de corpúsculos de Müller en los trichilia. Encontramos que la presencia de corpúsculos de Müller no afecta la actividad de las colonias y por tanto, esta tiene mayor relación al ritmo diario de la especie que a la presencia de alimento. En cuanto a los resultados a nivel general en las colonias en cautiverio la actividad mostró una tendencia mayor en las horas cercanas al mediodía, lo que sugiere una relación con el ciclo diario. La planta aumenta la producción de cuerpos Müller según aumenta la actividad de la hormiga. Además la relación de la hormiga con Pseudococidae pone en duda los beneficios que recibe la planta en su relación con Azteca. Finalmente, la actividad en las colonias en el campo varió mucho, lo que sugiere que durante estas observaciones no solo influyó el ritmo diario, sino que además hay un efecto de factores ambientales sobre la actividad de la hormiga.

PALABRAS CLAVE

Pseudococcidae, cuerpos de Müller, trichilium, ciclo diario, fotoperiodo
La actividad de las hormigas, al igual que en el resto de los seres vivos, varía de manera rítmica en fase a los ciclos de rotación de la Tierra y de su traslación alrededor del Sol. Esas variaciones rítmicas alcanzan los valores mínimos y máximos en distintas horas del ciclo, lo que sugiere un sistema cronobiológico interno integrado (Aréchiga, 2004). Según Aréchiga (2004) los ritmos biológicos de periodicidad diurna o estacional pueden persistir incluso en condiciones ambientales constantes, desde luego en condiciones normales los ritmos diarios están sincronizados por las variaciones geofísicas.

Estudios de campo y laboratorio en Tapinoma nigerrima y Crematogaster scutellaris han revelado que la actividad de las colonias de hormigas pueden variar de una estación a otra, además puede variar durante el día de una especie a otra (Redolfi, Tinaut, Pascual \& Campos, 2003). Por otro lado, se ha reportado que en Camponotus sp. los 
cambios de actividad no solamente se encuentran ligados al fotoperiodo, sino también a la oferta de alimento de la planta con la que se encuentra relacionada, presentando mayor actividad de la hormiga cuando la planta tiene mayor producción de néctar (Novoa, Redolfi \& Ceroni, 2005).

Muchas de las especies que conforman interacciones con plantas, constituyen una defensa contra posibles amenazas para estas e involucran desde sistemas obligados hasta relaciones difusas (Farji, Folgarait \& Protomastro, 1992). Hay aproximadamente 150 especies y subespecies de hormigas Azteca en el neotropico (Hogue, 1993; Longino, 1991b), y de éstas, en Costa Rica cinco especies viven y se alimentan en plantas de Cecropia (Longino, 1991a; Longino, 1996; Longino, 2007); en su mayoría estas plantas presentan una serie de caracteres que parecen adaptados específicamente para sostener colonias de hormigas (Longino, 1991a; Thompson, 2003), tales como las cavidades naturales del tronco y la formación de cuerpos alimenticios llamados corpúsculos de Müller en estructuras Ilamadas trichilium en la base del peciolo de las hojas, estos son ricos en glucógeno, lípidos y proteínas, y sirven de alimento para sus hospederos (Fernández, 2003).

El presente trabajo tiene como objetivos registrar los patrones de actividad de Azteca constructor en condiciones de cautiverio y en el campo. Determinar si la actividad diaria de Azteca varía ante la presencia de corpúsculos de Müller en el trichilium o si su actividad tiene mayor relación al ritmo circadiano de la especie que a la presencia de alimento. Evaluamos dos hipótesis, que la actividad de la hormiga Azteca constructor se relaciona en mayor medida con (1) la presencia de alimento en la planta y (2) que obedece a la hora del día.

\section{MATERIALES Y MÉTODOS}

El estudio de campo lo realizamos los días 5 y 26 de noviembre, 2012 en las Instalaciones Deportivas de la Universidad de Costa Rica, Sabanilla, Montes de Oca, San José (956'41"N 8402'47"W, $1224 \mathrm{msnm}$ ). El clima en esta zona del país es de tipo isotermal templado; fresco y seco, con temperatura promedio anual de 19 a $22^{\circ} \mathrm{C}$ y humedad relativa del 82\%. Reporta precipitación de 1 200-2 200mm anuales. El ecosistema natural pertenece al piso premontano, a la zona de vida bosque húmedo premontano, con un alto grado de alteración por urbanización (Álvarez \& Campos, 2012). Las observaciones en cautiverio las realizamos los días 6 de agosto y 1 de noviembre en Esquipulas de Palmares, Alajuela ( $10^{\circ} 3^{\prime} 31^{\prime \prime} \mathrm{N} 84^{\circ} 25^{\prime} 09^{\prime \prime} \mathrm{O}, 1$ 005msnm).

Evaluamos dos hipótesis, que la actividad de la hormiga Azteca constructor se relaciona en mayor medida con
(1) la presencia de alimento en la planta y (2) que obedece a la hora del día. Para ello localizamos cuatro colonias de Azteca constructor que interactuaban con individuos de Cecropia obtusifolia menores a $2 \mathrm{~m}$ de altura (plantas jóvenes). Capturamos dos colonias para trabajo en laboratorio (colonias 1 y 2) y dos para observaciones en el campo (colonias 3 y 4 ).

La captura de la colonia 1 se hizo el día 29 de septiembre y la captura de la colonia 2 se hizo el día 26 de noviembre. Para capturar las hormigas de ambas colonias, retiramos las hojas de la planta y procurando no dañar el nido cortando el tallo en trozos pequeños de $20 \mathrm{~cm}$ a $40 \mathrm{~cm}$, desde la copa hasta la base. Recolectamos los trozos de tallo dentro de una taza plástica marca Ziploc ${ }^{\circledR}$ de $45 \mathrm{~cm}$ de largo y $10 \mathrm{~cm}$ de alto. Atrapamos la mayor cantidad de hormigas posible, incluyendo las obreras, la reina, los huevos, las pupas y las larvas. Recolectamos los peciolos con trichilium en una bolsa con cierre hermético marca Ziploc $^{\circledR}$. Ambas colonias fueron trasladadas a Esquipulas de Palmares, donde colocamos los trozos de tallo de C. obtusifolia con la cámara real, las obreras y huevos capturados en una pecera de vidrio de $43 \mathrm{~cm}$ de largo por $30 \mathrm{~cm}$ de ancho y $13 \mathrm{~cm}$ de alto. La aclimatación al cautiverio de la colonia 1 se dio entre los días 30 de agosto y 5 de septiembre. El periodo de aclimatación de la colonia 2 se dio entre los días 27 y 30 de noviembre. Durante el periodo de aclimatación de las colonias estas no recibieron alimento.

Para medir el ciclo de actividad de las hormigas en cautiverio realizamos conteos de hormigas sobre los trozos del tallo que contenía la cámara real (área sobre el nido), sobre los trichilium y sobre otros sitios (cualquier otra zona dentro de la pecera) durante 24horas, dividiendo el día como se muestra en el Cuadro 1. Para los conteos en cautiverio realizamos conteos cada $15 \mathrm{~min}$, para un total de siete para cada sitio en cada momento del día; a nivel general se toma en cuenta 21 conteos por momento del día. Por otro lado para las observaciones en el campo se hicieron cada 10min, y con la misma cantidad de observaciones, se tomó en cuenta el área sobre el tallo de C. obtusifolia (área sobre el nido), sobre los trichilia y sobre otros sitios (hojas, peciolos). Esto se hizo durante 8 horas dividiendo el día como se muestra en el Cuadro 1. En cada observación en cautiverio, continuamente para determinar si la actividad varía como efecto de la presencia de corpúsculos de Müller, expusimos a las hormigas a dos tratamientos (con corpúsculos de Müller y sin corpúsculos de Müller), colocamos durante 3horas trichilia con corpúsculos de Müller y durante las tres horas siguientes trichilia sin estos, repitiendo esto durante toda la observación, para esto se tomó en cuenta 28 observaciones para cada sitio para un total de 84 observaciones por tratamiento a nivel general. 


\section{CUADRO 1}

Códigos y rangos de hora de cada momento del día para los conteos de Azteca constructor en cautiverio y en el campo.

\begin{tabular}{cccccc}
\hline & Cautiverio & \multicolumn{3}{c}{ Campo } \\
\hline Momentos & Códigos & Horas & Momentos & Códigos & Horas \\
\hline Madrugada & ma1 & 0:00-3:00 & Mañana & M1 & 8:00-10:00 \\
& ma2 & $3: 00-6: 00$ & & & \\
Mañana & mñ1 & $6: 00-9: 00$ & & M2 & $10: 00-12: 00$ \\
& mñ2 & $9: 00-12: 00$ & & & \\
\hline Tarde & $\mathrm{t} 1$ & $12: 00-15: 00$ & Tarde & T1 & $12: 00-14: 00$ \\
& $\mathrm{t} 2$ & $15: 00-18: 00$ & & & \\
Noche & $\mathrm{n} 1$ & $18: 00-21: 00$ & & T2 & $14: 00-16: 00$ \\
& $\mathrm{n} 2$ & $21: 00-00: 00$ & & &
\end{tabular}

Realizamos el análisis de datos en Excel 2007, el programa estadístico JMP (SAS Institute Inc, 2007) y el programa G-Stat 2.0. Definimos como regla de decisión un $\alpha=0.05$ y la desviación estándar como medida de variación. Utilizamos el método de números aleatorios de Microsoft Excel 2007 para definir la frecuencia de los conteos para cada sitio durante todas las observaciones. Con el fin detectar diferencias significativas en la actividad de las hormigas en los distintos momentos del día en cada sitio y a nivel general, y además para determinar el efecto en la actividad de las hormigas ante la presencia o ausencia de los cuerpos de Müller en los trichilia, realizamos la prueba no paramétricas de Kruskal-Wallis debido al incumplimiento del supuesto de normalidad de los datos (Diaz, 2009). En los casos en los que hubieron diferencias significativas se hizo una prueba post-hoc, en la que se hizo comparación múltiple de rangos promedio mediante el método de Dunn (Diaz, 2009).

\section{RESULTADOS}

En las observaciones en laboratorio la colonia 1 presentó el menor número promedio de individuos $(4,24 \pm 4,99)$ que la colonia $2(6,91 \pm 8,02)$. La colonia 3 presento menor número de individuos $(7,43 \pm 7,86)$ que la colonia 4 $(21,42 \pm 12,1)$.

En el conteo general de hormigas en la colonia 1 hubo diferencia significativas entre la actividad presente en los diferentes momentos del día $(H=37,7, g l=7, p<0,05)$; el momento 1 presento la mayor actividad; diferencias sólo en los momentos ma 1 con $\mathrm{t} 1$, ma 2 con $\mathrm{t} 1, \mathrm{mn} 1$ con $\mathrm{t} 1 \mathrm{y} t 2$ con mñ1 (Dunn, $p<0,05$, Fig. 1 A). La actividad sobre el nido presentó diferencias significativas entre los momentos del día $(\mathrm{H}=32,3, \mathrm{gl}=7, \mathrm{p}<0,05)$; el momento 1 presentó la mayor actividad; los momentos diferentes fueron ma2 con $\mathrm{t} 1$, mñ 1 con $\mathrm{t} 1, \mathrm{n} 1$ con mñ1 y $\mathrm{n} 2$ con mñ 1 (Dunn, $\mathrm{p}<0,05$, Fig.1B). La actividad sobre el trichilium presentó diferencias significativas entre los diferentes momentos del día $(H=25,6, g l=7, p<0,05)$; el momento $t 1$ presentó la mayor actividad; diferencias en los momentos mñ 1 con $t 2$ y mñ 1 con n2 (Dunn, $p<0,05$, Fig.1C). La actividad sobre otros sitios presentó diferencias significativas entre los diferentes momentos del día $(H=27,2, g l=7, p<0,05)$; la mayor actividad de las hormigas se observo en $\mathrm{t} 1$; las diferencias se observaron en los momentos $\mathrm{t} 1$ con ma1 y $\mathrm{t} 1$ con $\mathrm{n} 2$ (Dunn, $p<0,05$, Fig.1D).

En la colonia 2 en el conteo general no hubo diferencia significativas en la actividad presente en los diferentes momentos del día ( $H=9,42, g l=7, p=0,22$, Fig.2A). En cambio en la actividad sobre el nido hubo diferencia significativas entre los diferentes momentos del día $(H=46,0$, $\mathrm{gl}=7, \mathrm{p}<0,05)$; el momento $\mathrm{t} 1$ presentó la mayor actividad; los momentos diferentes fueron ma2 con mñ2, ma1 con $\mathrm{t} 1$, ma1 con $\mathrm{t} 2$, ma2 con $\mathrm{t} 1, \mathrm{t} 1$ con $\mathrm{n} 1$ y $\mathrm{t} 1$ con $\mathrm{n} 2$ (Dunn, $\mathrm{p}<0,05$, Fig.2B). La actividad sobre el trichilium presentó diferencia significativas entre los diferentes momentos del día $(H=24,1, g l=7, p<0,05)$; el momento mñ1 presentó la mayor actividad; diferencias en los momentos mñ1 con ma1 y mñ1 con t2 (Dunn, $p<0,05$, Fig.2C). Por otro lado la actividad sobre otros sitios no presento diferencia significativas entre los diferentes momentos del día $(H=8,60$, $g l=7, p=0,28$, Fig.2D). 
En la colonia 3, a nivel general no hubo diferencias significativas en la actividad de las hormigas durante los cuatro momentos del día ( $\mathrm{H}=5,59, \mathrm{gl}=3, \mathrm{p}=0,13$, Fig.3A). La actividad sobre el nido presentó diferencias significativas $(H=16,6, g l=3, p<0,05)$; el momento $M 2$ presento la mayor actividad de hormigas; las diferencias fueron en los momentos $\mathrm{M} 2$ con $\mathrm{M} 1$ y $\mathrm{T} 2$ con $\mathrm{M} 1$ (Dunn, $\mathrm{p}<0,05$, Fig.3B). La actividad sobre los trichilia presentó diferencias significativas $(H=9,35, g l=3, p<0,05)$; con la mayor actividad de hormigas en $\mathrm{T} 1$; $y$ diferencias en el momentos T1 con M1 (Dunn, $p<0,05$, Fig.3C). Además la actividad en otros sitios presentó diferencias significativas $(H=14,6, g l=3, p<0,05)$; la mayor actividad se observó en el momento $\mathrm{M} 2$; se observaron diferencias en $\mathrm{T} 2$ con M2 (Dunn, $p<0,05$, Fig.3D).

En la colonia 4 a nivel general hubo diferencias significativas en la actividad de las hormigas $(\mathrm{H}=8,46, \mathrm{gl}=3$, $\mathrm{p}<0,05)$; con la mayor actividad en $\mathrm{M} 1$; las diferencias se observaron en T2 con M1 (Dunn, p<0,05, Fig.4A). La actividad sobre el nido presentó diferencias significativas $(H=13,1, g l=3, p<0,05)$; la actividad en $M 1$ fue la más alta; y hubo diferencias en los momentos $\mathrm{M} 2$ con M1 y M1 con T2 (Dunn, p<0,05, Fig.4B). La actividad sobre los trichilia no presentó diferencias significativas durante los distintos momentos del día ( $H=1,35, g l=3, p=0,72$, Fig.4C). Por otro lado la actividad de las hormigas en otros sitios si presentó diferencias significativas $(H=16,7, g l=3, p<0,05)$. La mayor actividad se observó en el momento $\mathrm{M} 1$; las diferencias en los momentos fueron T2 con M1 (Dunn, $p<0,05$, Fig.4D).

Con respecto a la actividad ante la presencia o ausencia de cuerpos de Müller, no se encontró suficiente evidencia de diferencias significativas en la colonia 1 (Cuadro 2), a nivel general $(H=0,42, g l=1, p=0,51)$, ni en la actividad sobre el trichilium $(H=1,09, g l=1, p=0,29)$, ni en la actividad en los otros sitios $(H=0,01, g l=3, p=0,93)$, tampoco se observaron diferencias en la actividad sobre el nido $(H=0,03$, $g \mathrm{l}=1, \mathrm{p}=0,86$ ). Por otro lado en la colonia 2 (Cuadro 2), no hubo diferencias cuando hubo presencia o ausencia de los cuerpos de Müller en los trichilia en la actividad a nivel general $(H=0,78, g l=1, p=0,38)$, ni en la actividad sobre el trichilium $(\mathrm{H}=0.06, \mathrm{gl}=1, \mathrm{p}=0.81)$, ni en la actividad en los otros sitios $(H=0,24, g l=3, p=0,62)$, mientras que si observaron diferencias en la actividad sobre el nido con una mayor actividad cuando había presencia de cuerpos de Müller ( $H=4,64, g l=1, p=0,03)$.
A

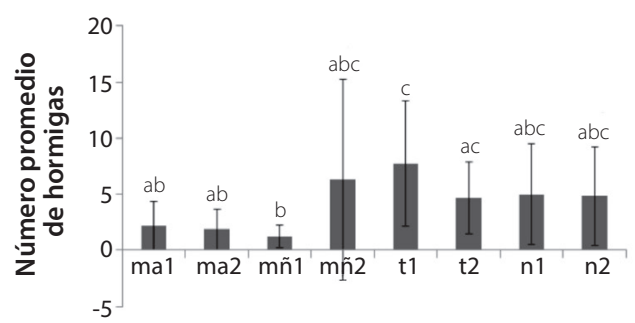

C

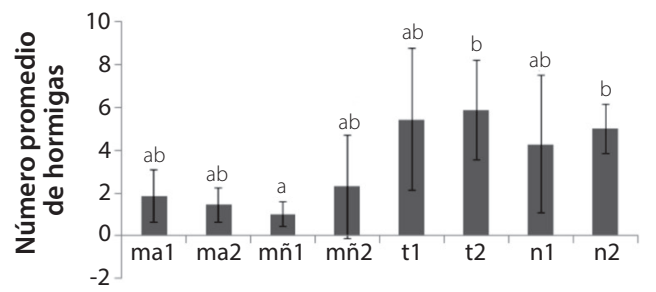

B

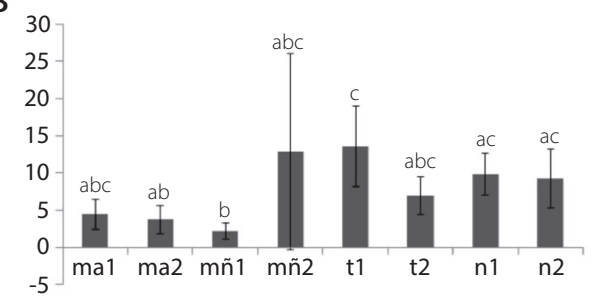

D

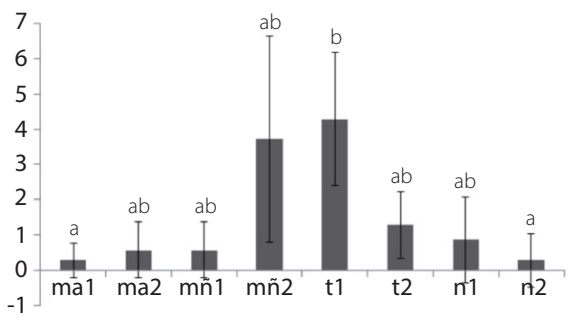

FIG.1. Promedios y desviación estándar del número de Azteca constructor en cada momento del día para la Colonia 1. (A) Actividad a nivel general $(H=37,7, g l=7, p<0,05)$. (B) Actividad sobre el nido $(H=32,3, g l=7, p<0,05)$. (C) Actividad sobre los trichilia $(H=25,6, g l=7, p<0,05)$. (D) Actividad sobre otros sitios $(H=27,2, g l=7, p<0,05)$. Para cada figura: $a, b, c$ representan agrupaciones distintas. Para categorías con más de una letra: la primera letra con similitudes a la categoría de cada letra adjunta (Dunn, $p<0,05)$. Categorías en rangos de hora: ma1: (00:00-02:45), ma2: (03:0006:00), mñ1: (06:00-09:00), mñ2: (09:00-12:00), t1: (12:00-15:00), t2: (15:00-18:00), n1: (18:00-21:00) y n 2: (21:00-00:00). 
A

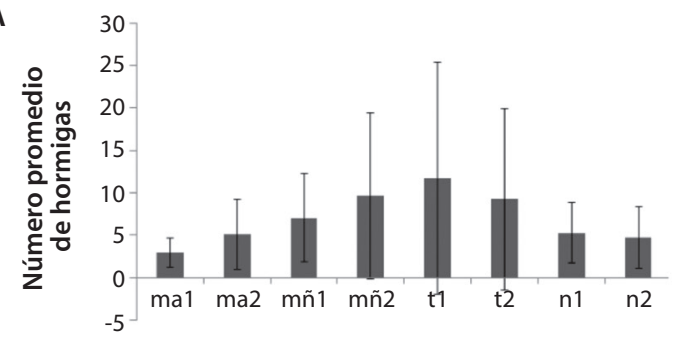

C

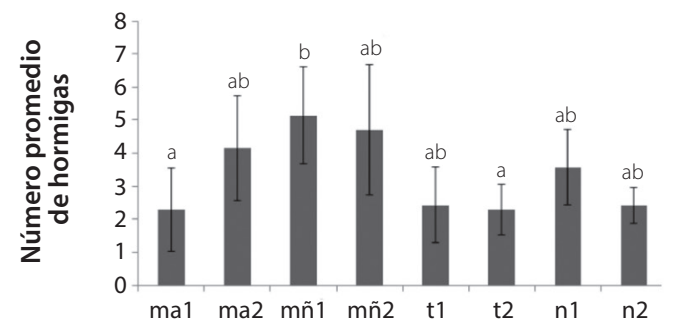

B

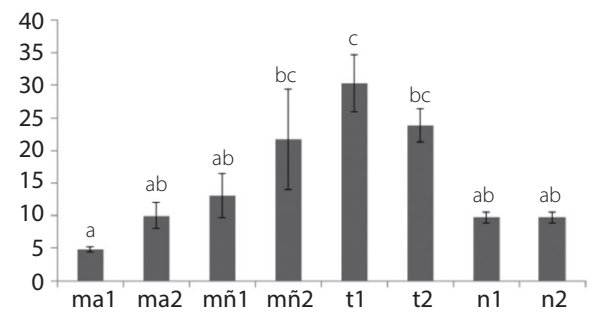

D

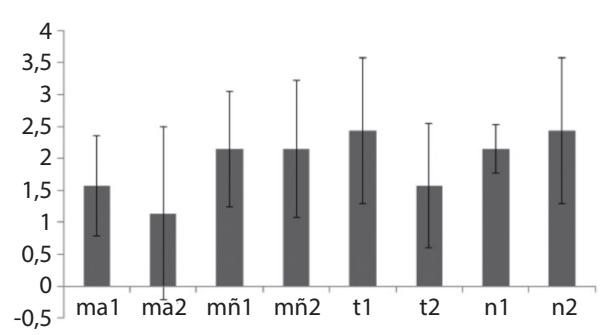

FIG. 2. Promedios y desviación estándar del número de Azteca constructor en cada momento del día para la Colonia 2. (A) Actividad a nivel general $(H=9,42, g l=7, p=0,22)$. (B) Actividad sobre el nido $(H=46,0, g l=7, p<0,05)$. (C) Actividad sobre los trichilia $(H=24,1, g l=7, p<0,05)$. (D) Actividad sobre otros sitios $(H=8,60, g l=7, p=0,28)$. Para cada figura: $a, b$, c representan agrupaciones distintas. Para categorías con más de una letra: la primera letra con similitudes a la categoría de cada letra adjunta (Dunn, $p<0,05)$. Categorías en rangos de hora: ma1: (00:00-02:45), ma2: (03:0006:00), mñ1: (06:00-09:00), mñ2: (09:00-12:00), t1: (12:00-15:00), t2: (15:00-18:00), n1: (18:00-21:00) y n2: (21:00-00:00).

A

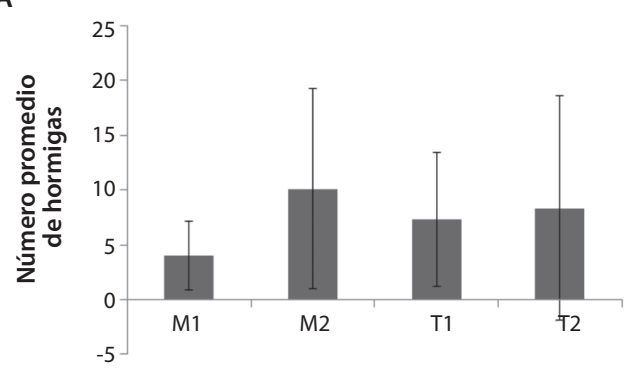

C

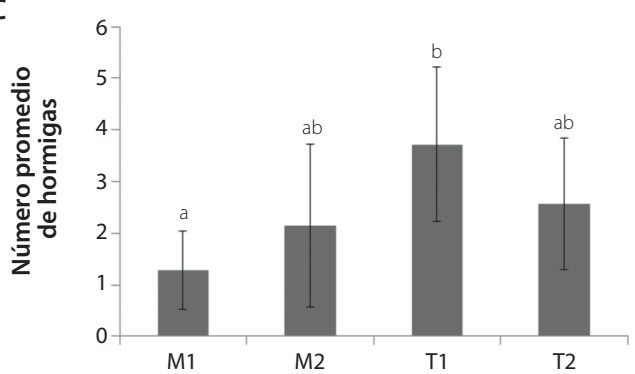

B

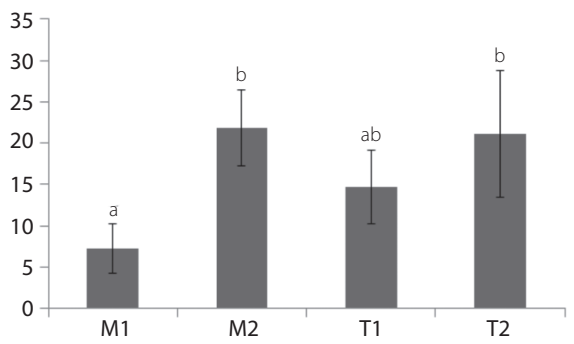

D

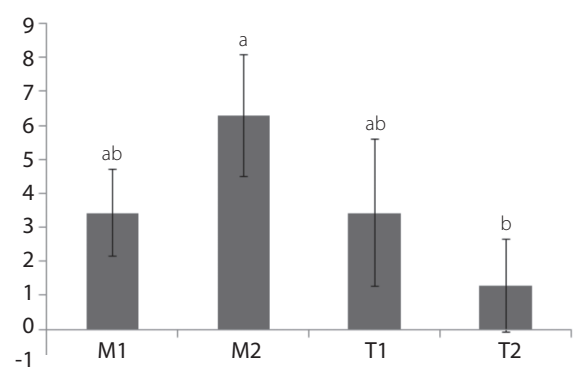

FIG. 3. Promedios y desviación estándar del numero de Azteca constructor en cada momento del día para la Colonia 3. (A) Actividad a nivel general $(H=5,59, g l=3, p=0,13)$. (B) Actividad sobre el nido $(H=16,6, g l=3, p<0,05)$. (C) Actividad sobre los trichilia $(H=9,35, g l=3, p<0,05)$. (D) Actividad sobre otros sitios $(H=14,6, g l=3, p<0,05)$. Para cada figura: $a, b, c$ representan agrupaciones distintas. Para categorías con más de una letra: la primera letra con similitudes a la categoría de cada letra adjunta (Dunn, $\mathrm{p}<0,05)$. Categorías en rangos de hora: M1: (08:00-10:00), M2: (10:00-12:00), T1: (12:00-14:00) y T2: (14:00-16:00). 
A

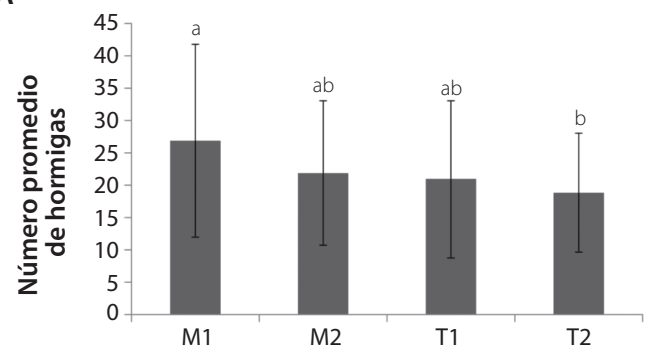

C



B

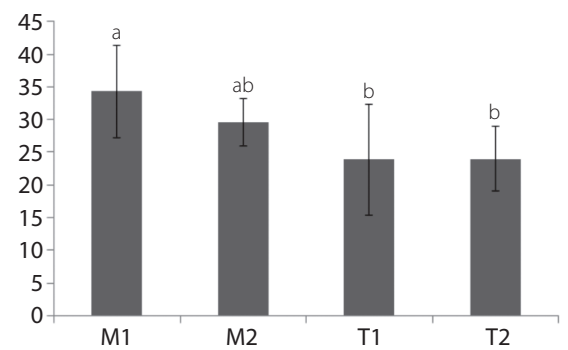

D

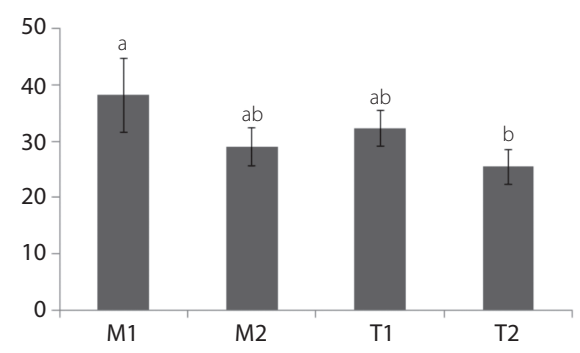

FIG. 4. Promedios y desviación estándar del numero de Azteca constructor en cada momento del día para la Colonia 4. (A) Actividad a nivel general $(H=8,46, g l=3, p<0,05)$. (B) Actividad sobre el nido $(H=13,1, g l=3, p<0,05)$. (C) Actividad sobre los trichilia $(H=1,35, g l=3, p=0,72)$. (D) Actividad sobre otros sitios $(H=16,7, g l=3, p<0,05$. Para cada figura: $a, b, c$ representan agrupaciones distintas. Para categorías con más de una letra: la primera letra con similitudes a la categoría de cada letra adjunta (Dunn, $\mathrm{p}<0,05)$. Categorías en rangos de hora: M1: (08:00-10:00), M2: (10:00-12:00), T1: (12:00-14:00) y T2: (14:00-16:00).

CUADRO 2

Promedios y desviación estándar del conteo de Azteca constructor en cada momento del día para los tratamientos: con presencia o en ausencia de cuerpos de Müller. Para cada colonia en cautiverio, a nivel general y en cada uno de los sitios.

\begin{tabular}{cccccc}
\hline & Variable & General & Sobre trichilium & Sobre Nido & Otros Sitios \\
\hline Colonia 1 & Con & $4,05 \pm 4,52$ & $3,14 \pm 2,90$ & $7,50 \pm 5,51$ & $1,50 \pm 2,01$ \\
& Sin & $4,44 \pm 5,46$ & $3,64 \pm 2,54$ & $8,21 \pm 7,49$ & $1,46 \pm 2,06$ \\
Colonia 2 & Con & $7,79 \pm 9,20$ & $3,43 \pm 1,69$ & $17,9 \pm 9,76$ & $2,03 \pm 1,23$ \\
& Sin & $6,02 \pm 6,57$ & $3,32 \pm 1,63$ & $12,9 \pm 7,40$ & $1,86 \pm 0,80$
\end{tabular}




\section{DISCUSIÓN}

La actividad de las hormigas en cautiverio en la colonia 1 coincide con lo esperado, con los promedios más elevados al medio día y en las primeras horas de la tarde y los más bajos en la noche y la madrugada, lo que sugiere una relación con los cambios ambientales que ocurren durante el día. Según Silva (2010) los cambios en la actividad en los seres vivos están estrictamente relacionado con variaciones ambientales, como cambios en la temperatura y la intensidad de luz; además se dice que la mayoría de los seres vivos presentan cambios cíclicos en relación a su ciclo diario.

La actividad en la colonia 2 coincide con lo esperado, y con lo observado en la colonia 1. La actividad no varió a nivel general y la actividad en los otros sitios no mostró variaciones. No obstante, sobre el nido se vio una actividad distinta con los picos más altos durante las primeras horas de la tarde lo que coincide con lo observado en la primera colonia, y se relaciona con la respuesta del ciclo diario a las variaciones ambientales; como aumento de luz y calor. (Silva, 2010). En el presente trabajo no tomamos mediciones de la temperatura, pero nuestras observaciones sobre el clima nos indican que estos picos de actividad ocurrieron simultáneos a las horas de mayor temperatura en los días de muestreo. La actividad sobre el trichilium en la colonia 2 mostró una reducción de la actividad en los momentos de la tarde. Esta disminución puede asociarse al clima, dado que por la tarde observamos una disminución notoria de la temperatura ambiente, y las condiciones de cautiverio que utilizamos no aislaban la colonia de la temperatura ambiente ni de la incidencia de luz. Esta reportado que el frío adormece a las hormigas, cuando las temperaturas bajan, el mayor número posible de obreras busca refugio dentro del nido y reanudan sus funciones cuando la temperatura aumenta (Novoa et al., 2005).

Los resultados en la colonia 3 reflejan una actividad constante por parte de las hormigas a nivel general y no presenta ningún pico máximo de actividad. El aumento en la actividad sobre el nido y en otros sitios durante la mañana y en las horas cercanas al mediodía, así como la mayor actividad en el trichilium durante la tarde y horas más cercanas al mediodía, son variaciones que responden a regulación del reloj biológico y dependen esencialmente a las oscilaciones rítmicas del ciclo de luz y oscuridad (Amir \& Stewart, 1996), además del efecto por parte de las condiciones ambientales como la temperatura, que se encuentra altamente relacionada a dicho ciclo (Silva, 2010), se sabe que la temperatura puede tener efecto en la actividad de la colonia, y que ese efecto puede variar de una especie a otra (Fernández, 2003; Vargas et al., 2006). Por tratarse de observaciones hechas en el campo, la actividad de la colonia puede tener variaciones debido a las distintas variables que no son posibles de controlar, como el viento que al mover la planta provoca una reacción en las hormigas, además de la presencia de depredadores como arañas o insectos que pretendan depredar la planta. Ante ambos disturbios $A$. constructor reacciona aumentando su número y volviéndose agresiva. Un simple movimiento en la planta puede desencadenar una reacción en toda la coIonia (Del Val \& Dirzo, 2004).

El que en la colonia 4 la actividad a nivel general y sobre el trichilium se mantuviera constante, mientras que sobre el nido y otros sitios la actividad fuera decreciendo durante los conteos, puede deberse a las condiciones ambientales de baja temperatura del día del conteo. Estas pueden hacer que la colonia reduzca las actividades fuera del nido. Manteniendo sólo las funciones vitales a la colonia en ese momento (Fernández, 2003), como la recolección de alimento y su respectivo patrullaje en los trichilia, posponiendo las demás actividades fuera del nido como el patrullaje en hojas y peciolos, reduciendo también la defensa del nido solo a casos necesarios.

La actividad no varió con la presencia de cuerpos de Müller. A diferencia de lo esperado, proveer o no corpusculos de Müller a las hormigas no alteró su actividad a nivel general. Se observó la recolección de cuerpos de Müller por parte de las hormigas de manera constante mientras estos estaban presentes. Consideramos importante denotar que las hormigas se mantuvieron en constante actividad sobre el trichilium aun cuando no había cuerpos de Müller presentes. Otros estudios han mostrado que la producción de los cuerpos de Müller por parte de la planta depende de la actividad de las hormigas lo que indica que la actividad de la hormiga no está dependiendo de la producción de la planta, sino que ocurre lo contrario. La producción de cuerpos Müller decrece si se restringe el acceso a los mismos por parte de las hormigas, adema la remoción de cuerpos de Müller resulta en un aumento en la producción de los mismos, lo que indica una vez más que es la hormiga la que dispone de los beneficios de la relación con la planta, además se considera la producción de los cuerpos nutritivos por la planta un proceso costoso, vale la pena producirlo cuando tienen la protección por parte de las hormigas, pero cuando no están presentes es mejor utilizar la energía en otros procesos (Neighbor, 2011).

Como observación adicional a nuestros resultados, consideramos importante de notar que a las colonias en cautiverio se les proporciono gotas de agua con azúcar y en ausencia de cuerpos de Müller, las vimos alimentarse de estas. Además, encontramos en los domacios de Cecropia obtusifolia, Pseudococidos como posible fuente secundaria de alimento. El hecho de criar dentro de los domacios 
de la planta Pseudococidos por parte de las hor $\neg$ migas pone en duda los beneficios que recibe la planta en dicha relación, ya que estos insectos pueden provocar el crecimiento y penetración de bacterias y hongos en las plantas hospederas, disminuyendo la fotosíntesis de la planta, eventualmente puede ser transmisoras de virus, e inyectar toxinas a las plantas, por lo que no se considera del todo beneficio para Cecropia obtusifolia la presencia de Azteca constructor (Ramos \& Serna, 2004).

Se considera que el hecho de que la actividad varié en relación al ritmo diario pone en duda el nivel de efectividad de las hormigas en cuanto a la protección hacia la planta, ya que hay momentos en los que la actividad de la hormiga disminuye en gran medida lo que podría beneficiar en algún grado a algún depredador oportunista, o algún depredador con mayor grado de especialización hacia la planta, hay que demarcar que en la plantas observadas siempre se observaron hojas con depredación, no obstante nunca se observó algún insecto diferente de las hormigas sobre las plantas en las observaciones en el campo, esto sugiere que la depredación podría estar sucediendo durante las noche, o que ocurra constantemente pero en un grado muy bajo y raro. Según Neighbor (2011) la relación entre Azteca y Cecropia obtusifolia es un mutualismo facultativo para reducir herbívora, además se ha demostrado que las plantas sin hormigas sufren mayor daño por herbívora que las que sí tienen hormigas. Es importante considerar que las observaciones de este estudio vienen de plantas jóvenes, y que en plantas de mayor tamaño donde los recursos son mayores el tamaño de la colonia iría en aumento, lo que provocaría una variación en su comportamiento de forrajeo y protección al nido (Fernández, 2003), estudios han mostrado que la abundancia de las obreras de cada casta responde a las necesidades y a la edad de la colonia, un aumento en el número de obreras mayores resulta fundamental para un funcionamiento efectivo de la colonia (Hölldobler \& Wilson, 1990).

Consideramos este estudio de importancia básica para trabajos posteriores y con este contribuimos al conocimiento científico de la biología de Azteca constructor y al conocimiento general del comportamiento de hormigas en su estado silvestre o en cautiverio en Costa Rica, dado que estudios similares no habían sido realizados antes en el país.

\section{AGRADECIMIENTOS}

Agradecemos a M.Sc. Eduardo Chacón Madrigal por su colaboración durante la realización de este estudio, así como a Geovanny Mora por la identificación taxonómica de la hormiga.

\section{REFERENCIAS}

Álvarez, M. \& Campos, M. (2012). Ecosistemas forestales: la capacidad del uso de la tierra. San José, Costa Rica: Centro Científico Tropical.

Amir, S. \& Stewart, J. (1996). Resetting of the Daily Clock by a Conditioned Stimulus. Nature, 379, 542-545.

Aréchiga, H. (2004). Sustrato neural de los ritmos biológicos. Mensaje Bioquímico, 28, 225-250.

Del Val, A. \& Dirzo, R. (2004). Mirmecofilia: Las plantas con ejército propio. Interciencia, 29(12), 673-679.

Diaz, A. (2009). Diseño estadístico de experimentos. 2ed. Universidad de Antioquia, Colombia.

Farji, A., Folgarait, P., \& Protomastro, J. (1992). Asociación entre el arbusto Capparis retusa (Capparidaceae) y las hormigas Camponotus blandus y Acromyrmex striatus (Hymenoptera: Formicidae). Revista Biologia Tropical, 40(3), 341-344.

Fernández, L. (2003). Introducción a las hormigas de la región Neotropical. Colombia: Instituto de Investigación de Recursos Biológicos Alexander von Humboldt

Hogue, C.L. (1993). Latin America Insects and Entomology. California, USA: Universidad de California Press.

Hölldobler, B. \& Wilson, E.O. (1990). The ants. USA: Harvard University Press.

Longino, J.T. (1991a). Azteca ants in Cecropia trees: taxonomy, colony structure, and behavior. (pp. 271-288) In Huxley, C.R. \& Cutler (eds). Ant-plant interactions. Oxford. United Kingdom: Oxford University Press.

Longino, J.T. (1991b). Taxonomy of the Cecropia-inhabiting Azteca ants. Journal of Natural History, 25, 1571-1602.

Longino, J.T. (1996). Taxonomic characterization of some livestem inhabiting Azteca (Hymenoptera: Formicidae) in Costa Rica, with special reference to the ants of Cordia (Boraginaceae) and Triplaris (Polygonaceae). Journal of Hymenoptera Research, 5, 131-156.

Longino, J.T. (2007). A taxonomic review of the genus Azteca (Hymenoptera: Formicidae) in Costa Rica and global revision of the aurita group. Zootaxa, 1491, 1-63.

Neighbor, J. (2011). Müllerian body production by Cecropia obtusifolia (Cecropiaceae) with and without Azteca ants. Monteverde Institute Costa Rica, Tropical Ecology Collection, Monteverde, Costa Rica. Recuperado de http://guides. lib.usf.edu/content.php?pid=86148\&sid $=1608457 \&$ doi $=\mathrm{M} 39-00275 \#$ doi $=$ M39-00275

Novoa. S., Redolfi, I. \& Ceroni, A. (2005). Patrones de Actividad diario de la hormiga Camponotus sp. en los botones florales del cactus Neoraimondia arequipensis subs. roseiflora (Werdermann \& Backeberg) Oztolaza. Ecologia Aplicada, $4(1 / 2), 77-81$.

Ramos, A.P. \& Serna, F.C. (2004). Coccoidea de Colombia, con énfasis en las cochinillas harinosas (Hemiptera: 
Pseudococcidae). Revista Facultad Nacional de Agronomía Medellin, 57(2), 2383-2412.

Redolfi, A., Tinaut, A., Pascual, F. \& Campos, M. (2003). Patrón de actividad de Tapinoma nigerrima (Nylanderia) y Crematogaster scutellaris (Oliver) (Hymenoptera, Formicidae) en el cultivo de olivo y en el laboratorio. Zoologica Baetica, 13/14, 37-55.
Silva, F. (2010). Trastornos del ritmo Diario del sueño: fisiopatología, clasificación y tratamientos. Revista Memoriza.com, 7, 1-13.

Thompson, J.N. (2003). El proceso coevolutivo. Fondo de Cultura Económica, México.

Vargas, G. A. (2006). Efecto del estrés fisiológico en dos especies de hormigas (Formicidae) propias de cafetales con y sin sombra. Revista Colombiana de Entomología, 32(1), 61-66. 
